\title{
NOTE ON THE GREATEST INTEGER FUNCTION
}

\author{
BY M. A. BASOCO
}

1. Introduction. In this note we wish to record certain finite sums involving the greatest integer function $E(x)$, which seem to be of some interest. Hermite* has shown that the generating function for $E(x)$ has the simple form,

$$
\frac{x^{b}}{(1-x)\left(1-x^{a}\right)}=\sum_{(n)} E\left(\frac{n+a-b}{a}\right) x^{n},
$$

where $a, b$ are positive integers. To him is due, likewise, the development

$$
\frac{x^{b}}{(1-x)\left(1+x^{a}\right)}=\sum_{(n)} E_{1}\left(\frac{n+a-b}{2 a}\right) x^{n},
$$

where

$$
E_{1}(x)=E(2 x)-2 E(x)=E\left(x+\frac{1}{2}\right)-E(x) .
$$

As indicated by Hermite, these developments used in conjunction with the expansions for certain theta quotients, yield results of interest in the theory of numbers. Of particular importance are his results expressing some of Kronecker's class number sums (which arise from certain theta constants of the third degree) in terms of $E(x)$.

In what follows we are concerned with the application of Hermite's device to the twelve Jacobian elliptic functions and to the logarithmic derivatives of the theta functions. The results implicit in these theta series are first elicited in a form which involves an arbitrary function, which, when specialized to be $x^{r}$, yields relations between the sum of the $r$ th powers of the divisors of the integer $n$ and sums of $E(x)$ for certain values of the argument. For the analytical form of the series here used we

* Hermite, Acta Mathematica, vol. 5, pp. 310-324; Oeuvres, vol. 4, pp. 151-159. See also E. T. Bell, Algebraic Arithmetic, Colloquium Publications of this Society, vol. 7, p. 138. 
refer to any standard treatise, ${ }^{*}$ the corresponding arithmetized form being readily derived from them. $\dagger$

2. The Functions $\phi_{j}, \sigma_{j}, \rho_{j}$. Denote by $F(x)$ any arbitrary function and let $m$ range over the odd numbers $1,3,5, \cdots$, while $n$ is unrestricted. We define the functions $\phi_{j}, \sigma_{j}, \rho_{j}$ as follows:

$$
\begin{aligned}
\phi_{1}(x, n) & =\sum F(\tau x), & & n=t \tau . \\
\phi_{2}(x, m) & =\sum(-1 / \tau) F(t x), & & m=t \tau . \\
\phi_{3}(x, n) & =\sum(-1 / \tau) F(2 t x), & & n=t \tau . \\
\sigma_{1}(x, n) & =\sum(-1)^{\delta} F(2 d x), & & n=d \delta . \\
\sigma_{2}(x, n) & =\sum(-1 / \tau) F(\tau x), & & n=d \tau . \\
\sigma_{3}(x, n) & =-\sum(-1)^{d+\delta} F(2 d x), & n & =d \delta . \\
\rho_{1}(x, n) & =\sum F(2 t x), & & n=t \tau . \\
\rho_{2}(x, n) & =\sum(-1)^{d} F(2 d x), & n & =d \delta . \\
\rho_{3}(x, n) & =\sum F(2 d x), & & n=d \delta .
\end{aligned}
$$

In each of the preceding, the summations range over the divisors $d, \delta, t, \tau$ of the fixed positive integers $n, m$, the integer $\tau$ always being odd, and $(-1 \mid h)=(-1)^{(h-1) / 2}$ if $h$ is odd.

3. Sums in Terms of $E(x)$. The following sums are deduced from the expansions of the functions

$$
\begin{array}{lll}
\text { sn } & \text { cn } & d n \\
\text { ns } & \text { cs } & d s \\
\text { nc } & \text { sc } & d c \\
\text { nd } & \text { sd } & \text { cd, }
\end{array}
$$

using Glaishier's notation for the Jacobian elliptic functions. In our formulas, $n$ is a fixed arbitrary positive integer, $\mu$ ranges over $1,3,5, \cdots$, while $k$ ranges over $1,2,3,4, \cdots, n$.

$$
\sum_{(\mu)} \phi_{1}(x, \mu)=\sum_{(\mu)} E\left(\frac{n+\mu}{2 \mu}\right) F(\mu x),
$$

* See, for example, Whittaker and Watson, Modern Analysis, 4th ed., pp. 510-512.

† See E. T. Bell, Messenger of Mathematics, March, 1924, pp. 169-172, $\S \S 4,14$. 


$$
\sum_{(\mu)} \phi_{2}(x, \mu)=\sum_{(\mu)} E_{1}\left(\frac{n+\mu}{4 \mu}\right) F(\mu x) .
$$

In the left side of these expressions, $\mu$ ranges up to $(n-1)$ or $n$ according as $n$ is even or odd.

$$
\begin{aligned}
\sum_{(k)} \phi_{3}(x, k) & =\sum_{(k)} E_{1}\left(\frac{n+k}{4 k}\right) F(2 k x), \\
\sum_{(k)} \phi_{1}(x, k) & =\sum_{(\mu)} E\left(\frac{n}{\mu}\right) F(\mu x), \\
\sum_{(k)}(-1)^{k-1} \phi_{1}(x, k) & =\sum_{(\mu)} E_{1}\left(\frac{n}{2 \mu}\right) F(\mu x), \\
\sum_{(k)}(-1)^{k-1} \sigma_{2}(x, k) & =\sum_{(\mu)}\left(\frac{-1}{\mu}\right) E_{1}\left(\frac{n}{2 \mu}\right) F(\mu x), \\
\sum_{(s)} \sigma_{1}\left(x, \frac{n-s+1}{2}\right) & =-\sum_{(k)} E_{1}\left(\frac{n}{4 k}\right) F(2 k x), \\
\sum_{(s)} \sigma_{3}\left(x, \frac{n-s+1}{2}\right) & =\sum_{(k)}(-1)^{k} E_{1}\left(\frac{n}{4 k}\right) F(2 k x) .
\end{aligned}
$$

In (19) and (20) the index $s$ ranges over such values of $s \leqq n$ as will make $n-s \equiv 1, \bmod 2$.

$$
\begin{aligned}
\sum_{(k)} \sigma_{2}(x, k) & =\sum_{(\mu)}\left(\frac{-1}{\mu}\right) E\left(\frac{n}{\mu}\right) F(\mu x), \\
\sum_{(k)}(-1)^{k-1} \phi_{3}(x, k) & =\sum_{(k)}(-1)^{k-1} E_{1}\left(\frac{n+k}{4 k}\right) F(2 k x), \\
\text { (23) } \sum_{(s)}(-1)^{(n-s) / 2} \phi_{1}(x, n-s+1) & =\sum_{(\mu)}\left(\frac{-1}{\mu}\right) E_{1}\left(\frac{n+\mu}{4 \mu}\right) F(\mu x), \\
\text { (24) } \sum_{(s)} \sigma_{2}(x, n-s+1) & =\sum_{(\mu)}\left(\frac{-1}{\mu}\right) E\left(\frac{n+\mu}{2 \mu}\right) F(\mu x) .
\end{aligned}
$$

In (23) and (24) the index $s$ ranges over such values of $s \leqq n$ as will make $n-s \equiv 0, \bmod 2$.

The next results are deduced from the expansions of the logarithmic derivatives of the four elliptic theta functions. 


$$
\begin{aligned}
\sum_{(k)} \rho_{1}(x, k) & =\sum_{(k)} E\left(\frac{n+k}{2 k}\right) F(2 k x), \\
\sum_{(k)}(-1)^{k-1} \rho_{1}(x, k) & =\sum_{(k)}(-1)^{k-1} E\left(\frac{n+k}{2 k}\right) F(2 k x), \\
\sum_{(s)} \rho_{2}\left(x, \frac{n-s+1}{2}\right) & =\sum_{(k)}(-1)^{k} E\left(\frac{n}{2 k}\right) F(2 k x), \\
\sum_{(s)} \rho_{3}\left(x, \frac{n-s+1}{2}\right) & =\sum_{(k)} E\left(\frac{n}{2 k}\right) F(2 k x) .
\end{aligned}
$$

In (27) and (28) the index $s$ ranges over such values of $s \leqq n$ as will make $n-s \equiv 1, \bmod 2$.

4. Sums of Powers of Divisors of $n$. If in the preceding formulas the function $F(x)$ is taken to be $x^{r}, r$ a positive integer, we immediately deduce the following, where the notation is as follows:

$\zeta_{r}(n)=$ sum of the $r$ th powers of the divisors of $n$.

$\zeta_{r}^{\prime}(n)=$ sum of the $r$ th powers of the odd divisors of $n$.

$\psi_{r}(n)=$ sum of the $r$ th powers of the divisors of $n$ whose conjugates are odd.

$\delta_{r}(n)=$ the excess of the sum of the $r$ th powers of the divisors of $n$ whose conjugates are of the form $4 k+1$ over the like sum for those whose conjugates are of the form $4 k+3$.

$\delta_{r}^{\prime}(n)=$ the excess of the sum of the $r$ th powers of the $(4 k+1)$ divisors of $n$ over the like sum of the $(4 k+3)$ divisors of $n$.

$\alpha_{r}(n)=$ the excess of the sum of the $r$ th powers of the divisors of $n$ whose conjugates are even over the like sum for those whose conjugates are odd.

$\beta_{r}^{\prime}(n)=$ the sum of the $r$ th powers of the even divisors of $n$ diminished by the like sum of the odd divisors of $n$.

$\beta_{r}(n)=\sum(-1)^{d+\delta} d^{r}$, the sum extending over all the divisors $d, \delta$ of $n$.

$$
\begin{aligned}
& \sum_{(\mu)} \zeta_{r}(\mu)=\sum_{(\mu)} E\left(\frac{n+\mu}{2 \mu}\right) \mu^{r}, \\
& \sum_{(\mu)} \delta_{r}(\mu)=\sum_{(\mu)} E_{1}\left(\frac{n+\mu}{4 \mu}\right) \mu^{r} .
\end{aligned}
$$


On the left side of these, $\mu$ ranges up to $(n-1)$ or $n$ according as $n$ is even or odd.

$$
\begin{aligned}
\sum_{(k)} \delta_{r}(k) & =\sum_{(k)} E_{1}\left(\frac{n+k}{4 k}\right) k^{r}, \\
\sum_{(k)} \zeta_{r}^{\prime}(k) & =\sum_{(\mu)} E\left(\frac{n}{\mu}\right) \mu^{r} \\
\sum_{(k)}(-1)^{k-1} \zeta_{r}^{\prime}(k) & =\sum_{(\mu)} E_{1}\left(\frac{n}{2 \mu}\right) \mu^{r} \\
\sum_{(k)}(-1)^{k-1} \delta_{r}(k) & =\sum_{(\mu)}\left(\frac{-1}{\mu}\right) E_{1}\left(\frac{n}{2 \mu}\right) \mu^{r}, \\
\sum_{(s)} \alpha_{r}\left(\frac{n-s+1}{2}\right) & =-\sum_{(k)} E\left(\frac{n}{4 k}\right) k^{r}, \\
\sum_{(s)} \beta_{r}\left(\frac{n-s+1}{2}\right) & =\sum_{(k)}(-1)^{k-1} E_{1}\left(\frac{n}{4 k}\right) k^{r} .
\end{aligned}
$$

In (35) and (36) the index $s$ ranges over such values of $s \leqq n$ as will make $n-s \equiv 1, \bmod 2$.

$$
\begin{aligned}
\sum_{(k)} \delta_{r}^{\prime}(k) & =\sum_{(\mu)}\left(\frac{-1}{\mu}\right) E\left(\frac{n}{\mu}\right) \mu^{r} \\
\sum_{(k)}(-1)^{k-1} \delta_{r}(k) & =\sum_{(k)}(-1)^{k-1} E_{1}\left(\frac{n+k}{4 k}\right) k^{r} \\
\sum_{(s)}(-1)^{(n-s) / 2} \zeta_{r}(n-s+1) & =\sum_{(\mu)}\left(\frac{-1}{\mu}\right) E_{1}\left(\frac{n+\mu}{4 \mu}\right) \mu^{r} \\
\sum_{(s)} \delta_{r}^{\prime}(n-s+1) & =\sum_{(\mu)}\left(\frac{-1}{\mu}\right) E\left(\frac{n+\mu}{2 \mu}\right) \mu^{r} .
\end{aligned}
$$

In (39) and (40) the index $s$ ranges over such values of $s \leqq n$ as will make $n-s \equiv 0, \bmod 2$.

$$
\begin{aligned}
\sum_{(k)} \psi_{r}(k) & =\sum_{(k)} E\left(\frac{n+k}{2 k}\right) k^{r}, \\
\sum_{(k)}(-1)^{k-1} \psi_{r}(k) & =\sum_{(k)}(-1)^{k-1} E\left(\frac{n+k}{2 k}\right) k^{r},
\end{aligned}
$$




$$
\begin{aligned}
\sum_{(s)} \beta_{r}^{\prime}\left(\frac{n-s+1}{2}\right) & =\sum_{(k)}(-1)^{k} E\left(\frac{n}{2 k}\right) k^{r}, \\
\sum_{(s)} \zeta_{r}\left(\frac{n-s+1}{2}\right) & =\sum_{(k)} E\left(\frac{n}{2 k}\right) k^{r} .
\end{aligned}
$$

In (43) and (44) the index $s$ ranges over such values of $s \leqq n$ as will make $n-s \equiv 1, \bmod 2$.

5. Conclusion. If in the expansion for $\mathrm{dn}$ we put $x=0$, we obtain

$$
\vartheta_{3}^{2}(0, q)-1=\sum_{n=1}^{\infty} q^{n} f(n)=4 \sum_{n=1}^{\infty} \frac{q^{n}}{1+q^{2 n}},
$$

where $f(n)$ denotes the number of solutions of the equation

$$
x^{2}+y^{2}=n, \quad x, y \lesseqgtr 0 .
$$

From this it follows easily that

$$
f(1)+f(2)+f(3)+\cdots+f(n)=4 \sum_{(k)} E_{1}\left(\frac{n+k}{4 k}\right),
$$

so that

$$
f(n)=4 \sum_{(k)}\left\{E_{1}\left(\frac{n+k}{4 k}\right)-E_{1}\left(\frac{n+k-1}{4 k}\right)\right\} .
$$

Hermite, in a similar manner, has shown that

$$
f(1)+f(2)+f(3)+\cdots+f(n)=4 \sum_{(\mu)}\left(\frac{-1}{\mu}\right) E\left(\frac{n}{\mu}\right) .
$$

Hence, we have

$$
\sum_{(k)} E_{1}\left(\frac{n+k}{4 k}\right)=\sum_{(\mu)}\left(\frac{-1}{\mu}\right) E\left(\frac{n}{\mu}\right) .
$$

In a similar manner,

$$
\begin{aligned}
\vartheta_{2}{ }^{2}\left(0, q^{4}\right)=\sum q^{n} g(n) & =4 \sum_{(\mu)} \frac{q^{2 \mu}}{1+q^{4 \mu}} \\
& =4 \sum_{(\mu)}\left(\frac{-1}{\mu}\right) \frac{q^{2 \mu}}{1-q^{4 \mu}}
\end{aligned}
$$


where $g(n)$ denotes the number of solutions of the equation (46) with $x, y$ odd and $\gtrless 0$. Noting that $g(n)$ is zero unless $n \equiv 2$, $\bmod 8$, we obtain

$$
\begin{aligned}
g(2) & +g(10)+\cdots+g(8 r+2) \\
& =4 \sum_{(k)} E_{1}\left(\frac{2 r+k}{4 k-2}\right)=4 \sum(-1)^{k-1} E\left(\frac{2 r+k}{2 k-1}\right),
\end{aligned}
$$

from which $g(8 r+2)$ could be obtained explicitly in terms of either $E(x)$ or $E_{1}(x)$.

Lastly, since

$$
\begin{aligned}
\vartheta_{0}{ }^{2}(0, q)-1 & =4 \sum_{(n)}^{\infty}(-1)^{n} \frac{q^{n}}{1+q^{2 n}} \\
& =-4 \sum_{(\mu)}\left(\frac{-1}{\mu}\right) \frac{q^{\mu}}{1+q^{\mu}},
\end{aligned}
$$

we obtain,

$$
\begin{aligned}
h(1) & +h(2)+h(3)+\cdots+h(n) \\
& =4 \sum_{(\mu)}\left(\frac{-1}{\mu}\right) E_{1}\left(\frac{n}{2 \mu}\right)=4 \sum_{(k)}(-1)^{k-1} E_{1}\left(\frac{n+k}{4 k}\right),
\end{aligned}
$$

where $h(n)$ denotes the excess of the number of solutions of (46) with $x, y$ of opposite parity over the like with $x, y$ of the same parity.

From (54) it follows that

$$
h(n)=4 \sum_{(\mu)}\left(\frac{-1}{\mu}\right)\left\{E_{1}\left(\frac{n}{2 \mu}\right)-E_{1}\left(\frac{n-1}{2 \mu}\right)\right\} .
$$

Finally, it may be noted that a great many similar results are implicit in the expansions of the functions $\operatorname{sn}^{k} u, \mathrm{cn}^{k} u, \ldots$. The complete set of developments for the cases $k=3,4$ have been given by the writer in a former paper.*

The University of Nebraska

* Tôhoku Mathematical Journal, vol. 35 (1932), pp. 35-42. 